\title{
Prediction of Volume Responsiveness by Stroke Volume Variation vs. Central Venous Pressure in Children Undergoing Fontan Operation
}

\section{Yun'an Song}

Shanghai Children's Medical Center

Huiyan Hou

Shanghai Children's Medical Center

Jie Bai

Shanghai Children's Medical Center

Hongbin Gu ( $\nabla$ floodgu@hotmail.com )

Research article

Keywords: Volume responsiveness, Stroke volume variation, Central venous pressure, Fontan operation, Children

Posted Date: April 29th, 2020

DOI: https://doi.org/10.21203/rs.3.rs-22897/v1

License: (c) (i) This work is licensed under a Creative Commons Attribution 4.0 International License. Read Full License 


\section{Abstract}

Background: Fontan operation is a palliative medical procedure performed on children with singleventricle defects. As postoperative success of the procedure largely depends on the preload volume, it is necessary to ensure the effective volume of systemic circulation by maintaining an appropriate pressure gradient between the systemic vein and the left atrium. However, there is a lack of effective indexes to evaluate volume responsiveness in Fontan patients. Stroke volume variation (SVV) is a dynamic hemodynamic parameter based on cardiopulmonary interaction in mechanical ventilation. This study is to validate the sensitivity and specificity of SVV vs. central venous pressure (CVP) in assessing volume responsiveness of Fontan patients.

Method: 64 children were included in this prospective study with single-ventricle who underwent modified Fontan operation between May 2018 and January 2020. Patients were given $10 \mathrm{ml} \cdot \mathrm{kg}^{-1}$ albumin for volume challenge within 10 min after CPB. Before and after volume challenge, the invasive arterial pressure module was connected to Mostcare $^{\text {TM }}$ equipment to collect SBP, MBP, DBP, SVV, Cl and SVRI dynamically in a time window of $30 \mathrm{~s}$ at a frequency of $1000 \mathrm{~Hz}$. According to the range of $\mathrm{Cl}$ change, patients with $\Delta \mathrm{Cl} \geq 15 \%$ were classified into response (R) group and patients with $\Delta \mathrm{Cl}<15 \%$ into nonresponse (NR) group. Using SVV and CVP as indicators, the ROC of the patients was established, and the AUC, diagnostic threshold, sensitivity and specificity were calculated.

Results: The 64 pediatric patients included with a mean age of $4.85 \pm 1.20$ years, a mean height of 98.00 $\pm 16.74 \mathrm{~cm}$ and a mean weight of $15.65 \pm 5.37 \mathrm{~kg}$. The SVV value was $17.15 \pm 3.97 \%$ and $13.45 \pm 2.45 \%$ before and after fluid challenge treatment $v s .18 .60 \pm 1.83 \mathrm{mmHg}$ and $20.20 \pm 2.39 \mathrm{mmHg}$ for CVP in responders. The AUC of SVV was 0.74 (95\% confidence interval [CI] $0.54-0.94, \mathrm{P}<0.05)$ and the cutoff value was $16 \%$, offering a sensitivity of $50 \%$ and a specificity of $91.7 \%$ vs. $0.70(95 \% \mathrm{Cl} 0.50-0.92, \mathrm{P}>$ $0.05), 19.5 \mathrm{mmHg}, 58 \%$ and $76 \%$ for CVP.

Conclusion: SVV exhibited a good predictive value for volume responsiveness in pediatric Fontan patients. Appropriate volume therapy according to SVV could improve the cardiac function of such patients.

Trial registration: This research was registered in Chinese Clinical Trail Registry on Jan 26, 2018. Registration number is ChiCTR1800014654.Registry URL is http://www.chictr.org.cn/showproj.aspx? proj=25019. This observational prospective study was approved by the Local Ethics Committee of Shanghai Children's Medical Center affiliated to Shanghai Jiao Tong University (SCMCIRB-K2017035)

\section{Background}

Fontan operation is a palliative medical procedure normally performed on children with birth defects of the heart, in which, the systemic blood flow of the heart is attached directly to the pulmonary artery. ${ }^{(1)}$ As there is a lack of the pumping function of the right atrium, the ideal systemic venous (pulmonary artery) pressure (SVP) should be maintained between $12 \otimes 15 \mathrm{mmHg}$ and left ventricular end-diastolic pressure 


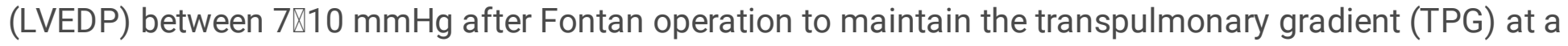
rational level of $2 \varangle 8 \mathrm{mmHg}$. In the process of anesthesia management, it is even more necessary to optimize intravascular hemodynamics, fluid volume, cardiac output and tissue perfusion. Unnecessary expansion of the preload volume may lead to adverse results and even increase mortality. ${ }^{(2)}$ In addition, some studies have pointed out that assessing the responsiveness of volume challenge can help distinguishing between postoperative cardiogenic and pulmonary circulatory failure, which is instructive for the subsequent treatment in Fontan patients. ${ }^{(3)}$ To the best of our knowledge, there has been less study reporting the assessment of volume responsiveness following Fontan operation. Although the reliability of stroke volume variation (SVV) in predicting fluid response in children with congenital heart disease such as ventricular septal defects has been well demonstrated, ${ }^{(4-6)}$ whether SVV can also be used in Fontan patients remains unclear. Compared with central venous pressure (CVP), the method and meaning of SVV calculation are different and therefore it is unclear whether SVV is suitable for patients with single ventricular circulation. In addition, there is little simple and effective golden standard method for prediction of volume responsiveness in pediatric patients. ${ }^{(7)}$ The aim of the present study is to determine whether SVV vs. CVP could accurately evaluate the volume responsiveness of children with Fontan circulation and provide evidence-based clues for clinically reasonable volume therapy in such patients.

\section{Methods}

\section{Patients}

This study was approved by the Medical Ethics Committee of Shanghai Children's Medical Center in Shanghai, China (SCMCIRB-K2017035) and certified by China clinical research registration (Registration No. ChiCTR1800014654). All parents or guardians of the patients voluntarily signed written informed consent before operation.

Included in this prospective single-center non-randomized study were 64 children with single-ventricle American Society of Anesthesiologists (ASA) physical status II-III who underwent modified Fontan operation under cardiopulmonary bypass (CPB) at Shanghai Children's Medical Center between May 2018 and January 2020. Written informed consent was obtained from the parents or guardians of all pediatric patients included. The inclusion criteria were children aged 3-8 years with ASA II or III who were scheduled for stage II modified Fontan operation with fenestration using cardiopulmonary bypass (CPB). The exclusion criteria were patients with arrhythmia before surgery whose mean pulmonary artery pressure was $\geq 18 \mathrm{mmhg}$, oxygen saturation was lower than $80 \%$, age was older than 9 years, aortic occlusion time was $>60 \mathrm{~min}$, and the positive inotropic agents score was $>10$ points. ${ }^{(8)}$ Volume challenge was observed in patients with sufficient hemostasis after CPB.

\section{Anesthesia management}


All patients were pre-medicated with oral midazolam $0.5 \mathrm{mg} \cdot \mathrm{kg}^{-1} 30 \mathrm{~min}$ before operation, and then induced with intravenous (IV) midazolam $0.1 \mathrm{mg} \cdot \mathrm{kg}^{-1}$, etomidate $0.3 \mathrm{mg} \cdot \mathrm{kg}^{-1}$, sufentanil $2 \mu \mathrm{g} \cdot \mathrm{kg}^{-1}$ and rocuronium $0.6 \mathrm{mg} \cdot \mathrm{kg}^{-1}$. Intubation was performed using a cuffed endotracheal tube. Mechanical ventilation was implemented by maintaining the pressure and keep the tidal volume at $10 \mathrm{ml} \cdot \mathrm{kg}^{-1}$, fraction of inspired oxygen at $50 \%$, the inspiratory to expiratory ratio at $1: 2$, and the respiratory rate at $14 \llbracket 20$ times per min to maintain $\mathrm{P}_{\mathrm{ET}} \mathrm{CO}_{2}$ at $30 \bigotimes 35 \mathrm{mmHg}$. Anesthesia was maintained by using propofol $4 \mathrm{mg} \cdot \mathrm{kg}^{-1} \cdot \mathrm{h}^{-1}$, sufentanil $2.5 \mu \mathrm{g} \cdot \mathrm{kg}^{-1} \cdot \mathrm{h}^{-1}$ and rocuronium $0.6 \mathrm{mg} \cdot \mathrm{kg}^{-1} \cdot \mathrm{h}^{-1}$. A $5.0 \mathrm{Fr}$ double-lumen catheter was set up in the right internal jugular vein for monitoring CVP and positive inotropic drug administration. A $20 \mathrm{Ga}$ catheter was retained in the left femoral vein for fluid infusion. A $22 \mathrm{Ga}$ catheter was instrumented in the left radial artery to allow for routine arterial pressure monitoring and advanced hemodynamic monitoring by Pressure Recording Analytical Method (PRAM) using Mostcare ${ }^{\text {TM }}$ device.

Before cardiac resuscitation, Dopamine $5 \mu \mathrm{g} \cdot \mathrm{kg}^{-1} \cdot \mathrm{min}^{-1}$ was administered, and the dose of positive inotropic agents was adjusted by the end of CPB using the maximal slope of systolic upstroke $\left(\mathrm{dp} / \mathrm{dt}_{\max }\right)$ monitored by PRAM and systolic arterial pressure. The dose of Dopamine was reduced at least to $1 \mu \mathrm{g} \cdot \mathrm{kg}^{-1} \cdot \mathrm{min}^{-1}$ in case $\mathrm{dp} / \mathrm{dt}_{\max }$ was $>1.2 \mathrm{mmHg} / \mathrm{ms}$ and systolic arterial pressure was $>100 \mathrm{mmHg}$. Additionally, Epinephrine was administered at $0.01 \llbracket 0.03 \mu \mathrm{g} \cdot \mathrm{kg}^{-1} \cdot \mathrm{min}^{-1}$ in case $\mathrm{dp} / \mathrm{dt}_{\max }$ was < $0.8 \mathrm{mmHg} / \mathrm{ms}$ and systolic arterial pressure was $<60 \mathrm{mmHg}$. Positive inotropic agents remained unchanged during the study period. After $\mathrm{CPB}$ and removal of the aortic cannulation, all patients received volume challenge with $5 \%$ albumin $10 \mathrm{ml} \cdot \mathrm{kg}^{-1}$ within $10 \mathrm{~min}$.

\section{PRAM and hemodynamic recording}

The standard arterial pressure transducer was routinely connected to the monitor using an anesthesia workstation (Datex-Ohmeda Aisys $\mathrm{CS}^{2}$, GE Healthcare, USA), which was also directly connected to the Mostcare $^{\text {TM }}$ to allow for transmission of the original signal and sampling at $1000 \mathrm{~Hz} .{ }^{(9)} \mathrm{SVV}$ was calculated simultaneously as the variation of beat-to-beat pulse pressure from the mean value during the most recent $30 \mathrm{~s}$ data and was displayed continuously using the following equation:

$$
S V V(\%)=\frac{\left(S V_{\max }-S V_{\min }\right)}{\left(S V_{\max }+S V_{\min }\right) / 2}
$$

Detailed 2-min measurements at $30 \mathrm{~s}$ intervals of each parameter were recorded by Mostcare ${ }^{\mathrm{TM}}$ and then downloaded to Excel sheets for offline analysis. Subsequently, the four consecutive measurements were averaged and adopted before or after volume challenge.

\section{Study protocol}

This study was designed to evaluate the accuracy of SVV in predicting fluid responsiveness in pediatric patients undergoing Fontan operation. They received fluid challenge with $5 \%$ albumin at $10 \mathrm{ml} \cdot \mathrm{kg}^{-1}$ for 10 minutes. Patients were defined as "responders" if the cardiac index increased more than $15 \%$ after a fluid 
infusion (group R), or "non-responders" if the cardiac index increase less than 15\% (groups NR). ${ }^{(10)}$ The medications remained unchanged during the study period. SVV was recorded by PRAM along with systolic and diastolic blood pressure, heart rate, stroke volume index, cardiac index, and CVP before and after fluid challenge.

\section{Statistical analysis}

Data are described as the mean \pm standard deviation (SD). The clinical continuous and categorical data between R and NR groups were compared by Student-t test and Fisher exact probability test respectively. Differences in hemodynamic parameters before and after volume challenge between group $\mathrm{R}$ and NR were examined by Student- $t$ test. Hemodynamic parameters before and after volume challenge within group R or group NR were constructed by paired $t$ test. ROC curves were established to assess the capacity of SVV and CVP for predicting fluid responsiveness. The optimal cutoff was confirmed when the sum of sensitivity and specificity was maximal. Statistical data were computed using SPSS 20.0 (IBM Corp, Armonk, NY, USA). ROC curves were mapped using Sigmaplot 12.0 (Systat Software Inc, San Jose, CA, USA).

\section{Results}

No significant adverse event occurred in any of the 64 Fontan patients successfully included in this study during the study protocol. Among them, 30 were responders and the remaining 34 were non-responders. The clinical data of all included patients are shown in Table 1. There was no significant difference in gender, age, height or weight between the two groups $(p>0.05)$.

Table 1

Clinical data of 64 Fontan patients in responders and non-responders

\begin{tabular}{|lllll|}
\hline Group & Gender M/F $(\mathbf{n})$ & Age $(\mathbf{y})$ & Height $(\mathbf{c m})$ & Weight $(\mathbf{k g})$ \\
\hline $\mathrm{R}$ & $30(18 / 12)$ & $4.97 \pm 1.14$ & $98.76 \pm 16.54$ & $15.84 \pm 5.69$ \\
\hline NR & $34(19 / 15)$ & $4.87 \pm 1.27$ & $97.73 \pm 17.43$ & $15.64 \pm 5.98$ \\
\hline Total & $64(37 / 27)$ & $4.85 \pm 1.20$ & $98.00 \pm 16.74$ & $15.65 \pm 5.37$ \\
\hline \multicolumn{4}{|l}{ Data is shown as $( \pm$ SD). There was no significant difference between the two groups. } \\
\hline
\end{tabular}

The hemodynamic parameters before and after volume challenge in group $\mathrm{R}$ and NR are shown in Table 2. There were significant differences in hemodynamic parameters (SBP, DBP, MBP, SVV, Cl and CVP) before and after volume challenge in group $\mathrm{R}$, in which $\mathrm{Cl}$ was $2.27 \pm 1.04 \mathrm{~L} \cdot \mathrm{min}^{-1} \cdot \mathrm{m}^{-2}$ and $2.76 \pm$ $1.09 \mathrm{~L} \cdot \mathrm{min}^{-1} \cdot \mathrm{m}^{-2}$ before and after volume challenge $v s .2 .00 \pm 1.17 \mathrm{~L} \cdot \mathrm{min}^{-1} \cdot \mathrm{m}^{-2}$ and $2.18 \pm 1.02 \mathrm{~L} \cdot \mathrm{min}^{-}$

$1 \cdot \mathrm{m}^{-2}$ in group NR, respectively. After volume challenge, CVP was $20.20 \pm 2.39 \mathrm{mmHg}$ in Group R vs. $19.64 \pm 3.83 \mathrm{mmHg}$ in group NR, showing no significant difference $(p>0.05)$. SVV in group $R$ was significantly lower than that in group NR $(13.45 \pm 2.45$ vs.14.91 $\pm 2.21, \mathrm{P}<0.05)$ after volume challenge. 
Table 2

Hemodynamic parameters recorded before and after volume challenge in responders and non-responders undergoing Fontan operation

\begin{tabular}{|c|c|c|c|}
\hline Parameter & Group & Before volume challenge & After volume challenge \\
\hline \multirow[t]{2}{*}{$\mathrm{HR}$ (beats. $\min ^{-1}$ ) } & $\mathrm{R}$ & $117 \pm 16.89$ & $121.61 \pm 15.93$ \\
\hline & NR & $124.5 \pm 13.64$ & $122.73 \pm 12.12$ \\
\hline \multirow[t]{2}{*}{$\mathrm{SBP}(\mathrm{mmHg})$} & $\mathrm{R}$ & $96.7 \pm 12.32$ & $102.4 \pm 10.00^{a}$ \\
\hline & NR & $95.71 \pm 11.11^{b}$ & $94.85 \pm 13.83$ \\
\hline \multirow[t]{2}{*}{$\mathrm{DBP}(\mathrm{mmHg})$} & $\mathrm{R}$ & $48.4 \pm 7.91$ & $59.3 \pm 7.25^{a}$ \\
\hline & NR & $58.07 \pm 7.80^{b}$ & $58 \pm 10.67$ \\
\hline \multirow[t]{2}{*}{$\mathrm{MBP}(\mathrm{mmHg})$} & $\mathrm{R}$ & $62.4 \pm 8.23$ & $75.3 \pm 6.85^{a}$ \\
\hline & NR & $72.07 \pm 7.51^{b}$ & $70 \pm 8.05$ \\
\hline \multirow[t]{2}{*}{ SVV (\%) } & $\mathrm{R}$ & $17.15 \pm 3.97$ & $13.45 \pm 2.45^{\mathrm{a}}$ \\
\hline & NR & $15.75 \pm 2.67^{b}$ & $14.91 \pm 2.21^{\mathrm{b}}$ \\
\hline \multirow[t]{2}{*}{ SVI $\left(m l \cdot m^{-2}\right)$} & $\mathrm{R}$ & $31.49 \pm 5.88$ & $37.51 \pm 4.84$ \\
\hline & NR & $33.70 \pm 6.20$ & $32.62 \pm 5.41$ \\
\hline \multirow[t]{2}{*}{$\mathrm{Cl}\left(\mathrm{L} \cdot \mathrm{min}^{-1} \cdot \mathrm{m}^{-2}\right)$} & $\mathrm{R}$ & $2.27 \pm 1.04$ & $2.76 \pm 1.09^{a}$ \\
\hline & NR & $2.00 \pm 1.17$ & $2.18 \pm 1.02$ \\
\hline \multirow[t]{2}{*}{ CVP (mmHg) } & $\mathrm{R}$ & $18.60 \pm 1.83$ & $20.20 \pm 2.39^{a}$ \\
\hline & NR & $18.57 \pm 2.34$ & $19.64 \pm 3.83$ \\
\hline \multicolumn{4}{|c|}{ Data is shown as $( \pm S D)$} \\
\hline \multicolumn{4}{|c|}{$\begin{array}{l}\text { HR heart rate; SBP systolic blood pressure; DBP diastolic blood pressure; MBP mean blood pressure; } \\
\text { SVV stroke volume variation; SVI stroke volume index; Cl cardiac index; CVP central venous pressure; } \\
\text { R responders; NR non-responders. }\end{array}$} \\
\hline \multicolumn{4}{|c|}{ a $P<0.05$ compared with that before volume challenge; } \\
\hline
\end{tabular}

There were significant differences in SBP, DBP, MBP, Cl and CVP before and after volume challenge in Group R (all $p<0.05)$, while no significant difference was observed in these parameters in group NR ( $p>$ 0.05). SBP, DBP and MBP values were significantly different between group $R$ and NR before volume challenge $(p<0.05)$. The value of SVV in group NR was significantly different from that in group $R$ both 
before and after volume challenge $(p<0.05)$. In addition, SVV in group R was decreased significantly after volume challenge $(p<0.05)$.

The AUC of SVV was $0.74(95 \% \mathrm{Cl} 0.54-0.94, \mathrm{P}<0.05)$ and the cutoff value was $16 \%$, offering a sensitivity of $50 \%$ and a specificity of $91.7 \%$ (Fig. 1). The AUC of CVP was 0.70 ( $95 \% \mathrm{Cl} 0.50-0.92, \mathrm{P}>$ 0.05 ) and the cutoff value was $19.5 \mathrm{mmHg}$, offering a sensitivity of $58 \%$ and a specificity of $76 \%$ (Fig. 2).

SBP, DBP, MDP, SVV, Cl and CVP values were significantly different before and after volume challenge in group R. SBP, DBP, MBP and SVV values were significantly different between responders and nonresponders before volume challenge. SVV was significantly different between responders and nonresponders after volume challenge.

\section{Discussion}

PRAM is a method for monitoring continuous cardiac output based on changes in arterial pressure, which is based on radial expansion caused by changes in volume of a given blood vessel. Many studies have demonstrated a good correlation of PRAM with other classical methods such as cardiac catheterization and Doppler ultrasound. ${ }^{(11,12)}$ SVV is a dynamic hemodynamic parameter that reflects changes in stroke volume. ${ }^{(13-15)}$ SVV in patients with mechanical ventilation is less than $10 \otimes 15 \%$. According to cardiopulmonary interaction under mechanical ventilation and Frank-Starling principle, a change in stroke volume caused by mechanical ventilation is more significant when the blood volume is insufficient, showing a negative correlation between SVV and the blood volume. ${ }^{(16,17)}$ Theoretically, SVV can therefore be used to estimate the intravascular volume state and predict the responsiveness of the circulatory system to the infusion treatment.

SVV has been used in predicting volume response in children receiving cardiac surgery. Some studies have shown that its accuracy is higher than CVP. $(18,19)$ To the best of our knowledge, this is the first study that demonstrated the good predictability of SVV for assessing fluid responsiveness after volume challenge in pediatric Fontan patients, proving that SVV is a simple, fast, direct and noninvasive method with good reproducibility. SVV can be measured with high-quality values in any individual patients. In this study, we implemented aggressive volume therapy by infusing $10 \mathrm{ml} \cdot \mathrm{kg}^{-1} 5 \%$ albumin instead of crystalloid solution within 10 minutes. $(20,21)$ The result of our experiment demonstrated that the hemodynamic parameters including the mean arterial pressure and cardiac index were significantly improved in the Fontan patients after volume challenge, as represented by a stable hemodynamic state, a balanced internal environment, an increase in urine volume, and the absence of significant adverse events. SVV $<16 \%$ indicated an insufficient blood volume of the patient and continuous volume expansion treatment had little effect in further improving the cardiac function in such a condition. Therefore, we believe that an appropriate volume and fluid type are primarily important for improving the circulation capacity and cardiac function of Fontan patients after operation. This may be related to the increase in TPG and pulmonary forward blood flow, though it needs to be verified in future research. 
The lung and blood vessel wall compliance in children is better than that in adults. However, the lung of children with a Fontan procedure is pathologically different from that of people with a biventricular structure. ${ }^{(22)}$ The higher positive airway pressure caused by mechanical ventilation will significantly reduce pulmonary blood flow and LVED volume. As Fontan patients have no normal right atrial and right ventricular structures in whom pulmonary vascular resistance (PVR) is relatively high, SVV produced by cardiopulmonary interaction may be significantly different between Fontan and normal children.

Currently, CVP is commonly used as a static hemodynamic parameter in clinical practice. However, some studies have demonstrated that CVP could not be used as a single reference in patients with volume therapy. ${ }^{(23)}$ As the linear relationship between cardiac pressure and volume is weakened in Fontan patients, the pressure parameter such as CVP cannot really reflect the volume state. In addition, the superior vena cava of Fontan patients is connected to the pulmonary artery, and therefore CVP is significantly affected by pulmonary artery pressure. As a result, the value of CVP to predict the volume reactivity of Fontan patients could be influenced by many factors and the result is unreliable.

There is few studies reporting the application of SVV in children with single-ventricle Fontan circulation. In this study, we found that the cutoff of SVV was $16 \%$, the sensitivity was $50 \%$, and the specificity was $91.7 \%$, suggesting that SVV is more specific than CVP in reflecting the volume state of children undergoing Fontan operation after CPB. Statistically, children with less SVV variation are more likely to be in a non-response state. Yoshitake et al ${ }^{(24)}$ used noninvasive hemodynamic monitoring to evaluate the cardiac output after Fontan operation by measuring the parameters of cardiac function in the patients with single left ventricular (SLV) and single right ventricular (SRV). Their results showed that the mean SVV (SLV : SRV) was $13.9 \%$ : $15.5 \%$, which is similar to our study. However, the sensitivity and specificity of SVV in our study suggest that SVV may have a high false-positive rate as a predictor of capacity reactivity in Fontan patients. This may be due to the more significant reduction of pulmonary blood flow in patients with single ventricle caused by positive pressure mechanical ventilation. In addition, the inhibition of myocardial contractility and the use of positive inotropic agents in early postoperative patients may affect the results. We believe that SVV derived from the expansion of the PPV index is a relatively safe volume reactivity parameter for patients who are not suitable to use volume dilation treatment, and therefore more suitable for patients whose current volume state is on the reaction section (steep section) of Frank-Starling curve rather than the smooth section. As each inspiratory and expiratory during mechanical ventilation can cause change in stroke volume, SVV can be used to indicate volume responsiveness and the current volume state of the patient.

This study has some limitations. First, the response of Fontan patients to volume infusion is affected by many factors. But as we only observed the effect of volume challenge alone, the influence of mechanical ventilation on the research results could not be excluded. In addition, we only observed the result of volume response immediately after volume challenge treatment without tracking changes in dynamic parameters in the cardiac intensive care unit. Finally, we did not use PiCCO as the control parameter for cost consideration. 


\section{Conclusion}

In summary, SVV has a good value for predicting changes in volume responsiveness in pediatric Fontan patients, although it may be affected by respiration and special physiological and anatomical structures. Appropriate volume challenge therapy can significantly improve the hemodynamic of patients and increase the cardiac output, devoid of significant impact on the internal environment. We believe that SVV can be used as a monitoring indicator in carrying out appropriate volume challenge treatment in pediatric Fontan patients, knowing that it can positively improve the cardiac function of the patients.

\section{Abbreviations}

SVV

Stroke volume variation; CVP:Central venous pressure; ROC:Receiver operating characteristic; AUC:Area under curve; Cl:Cardiac index; HR:Heart rate; SBP:systolic blood pressure; DBP:diastolic blood pressure; MDP:Mean blood pressure; SVI:Stroke volume index; CPB:cardiopulmonary bypass; TPG:Transpulmonary gradient

\section{Declarations}

\section{Funding}

This research was supported by the Young Scholar Research Grant from Shanghai Chilren's Medical Center (grant number: YJ-SCMC-2017-13).

\section{Authors' contributions}

This study was accomplished by four co-authors. The contributions of each author are as follows: YAS was involved in designing the study, collecting most of the data and wrote the original manuscript; $\mathrm{HYH}$ participated in the designing the study protocol and assisted YAS in collection of clinical data and Statistical analysis; JB and JJZ gave some instructions in designing the study scheme and performed general anesthesia management; HBG gave a lot of guidance to the design and writing of the manuscript. All authors read and approved the final manuscript.

\section{Author's information}

${ }^{1}$ Department of Anesthesiology, Shanghai Children's Medical Center, Shanghai Jiao Tong University School of Medicine \& National Children's Medical Center (Shanghai), 1678 Dongfang Road, Shanghai 200127, China.

${ }^{2}$ Pediatric Clinical Pharmacology Laboratory, Shanghai Children's Medical Center, Shanghai Jiao Tong University School of Medicine \& National Children's Medical Center (Shanghai), Shanghai, China.

\section{Acknowledgements}


We would like to express my sincere gratitude to all the patients and their parents for complete understanding and support to this research. Without them we would never been able to complete this important work. We also appreciate the efforts of all colleagues from the Department of Anesthesiology in Shanghai Children's Medical Center for supporting the research.

\section{Ethics approval and consent to participate}

This observational prospective study was approved by the Local Ethics Committee of Shanghai Children's Medical Center affiliated to Shanghai Jiao Tong University (SCMCIRB-K2017035) and all parents or guardians of the patients voluntarily signed written informed consent before operation.

Consent for publication: Not applicable

Availability of data and material: We have included the questionnaire used in this study as supplementary information. The datasets used and analysed during the current study are available from the corresponding author upon request.

Competing interests: All authors declare no competing interests.

\section{References}

1. Schäfer M, Frank BS, Humphries SM, Hunter KS, Carmody KL, Jacobsen R, et al. Flow Profile Characteristics in Fontan Circulation are Associated with the Single Ventricle Dilation and Function: Principal Component Analysis Study. Am J Physiol Heart Circ Physiol. 2020.

2. Wang X, Jiang L, Liu S, Ge Y, Gao J. Value of respiratory variation of aortic peak velocity in predicting children receiving mechanical ventilation: a systematic review and meta-analysis. Crit Care. 2019;23(1):372.

3. De Mey W, Cools B, Heying R, Budts W, Louw JJ, Boshoff DE, et al. Can a volume challenge pinpoint the limiting factor in a Fontan circulation? Acta Cardiol. 2015;70(5):536-42.

4. Favia I, Romagnoli S, Di Chiara L, Ricci Z. Predicting Fluid Responsiveness in Children Undergoing Cardiac Surgery After Cardiopulmonary Bypass. Pediatr Cardiol. 2017;38(4):787-93.

5. Lee JH, No HJ, Song IK, Kim HS, Kim CS, Kim JT. Prediction of fluid responsiveness using a noninvasive cardiac output monitor in children undergoing cardiac surgery. $\mathrm{Br} \mathrm{J}$ Anaesth. 2015;115(1):38-44.

6. Yi L, Liu Z, Qiao L, Wan C, Mu D. Does stroke volume variation predict fluid responsiveness in children: A systematic review and meta-analysis. PLoS One. 2017;12(5):e0177590.

7. Vistisen ST, Berg JM, Boekel MF, Modestini M, Bergman R, Jainandunsing JS, et al. Using extra systoles and the micro-fluid challenge to predict fluid responsiveness during cardiac surgery. J Clin Monit Comput. 2019;33(5):777-86.

8. Favia I, Rizza A, Garisto C, Haiberger R, Di Chiara L, Romagnoli S, et al. Cardiac index assessment by the pressure recording analytical method in infants after paediatric cardiac surgery: a pilot 
retrospective study. Interact Cardiovasc Thorac Surg. 2016;23(6):919-23.

9. Scolletta S, Romano SM, Biagioli B, Capannini G, Giomarelli P. Pressure recording analytical method (PRAM) for measurement of cardiac output during various haemodynamic states. $\mathrm{Br} \mathrm{J}$ Anaesth. 2005;95(2):159-65.

10. Ricci Z, Polito A, Netto R, De Razza F, Favia I, Carotti A, et al. Assessment of Modified Ultrafiltration Hemodynamic Impact by Pressure Recording Analytical Method During Pediatric Cardiac Surgery. Pediatric Critical Care Medicine. 2013;14(4):390-5.

11. Alonso-Inigo JM, Escriba FJ, Carrasco JI, Fas MJ, Argente P, Galvis JM, et al. Measuring cardiac output in children undergoing cardiac catheterization: comparison between the Fick method and PRAM (pressure recording analytical method). Paediatr Anaesth. 2016;26(11):1097-105.

12. Calamandrei M, Mirabile L, Muschetta S, Gensini GF, De Simone L, Romano SM. Assessment of cardiac output in children: A comparison between the pressure recording analytical method and Doppler echocardiography*. Pediatric Critical Care Medicine. 2008;9(3):310-2.

13. Guinot PG, de Broca B, Abou Arab O, Diouf M, Badoux L, Bernard E, et al. Ability of stroke volume variation measured by oesophageal Doppler monitoring to predict fluid responsiveness during surgery. Br J Anaesth. 2013;110(1):28-33.

14. Lee JY, Kim JY, Choi CH, Kim HS, Lee KC, Kwak HJ. The ability of stroke volume variation measured by a noninvasive cardiac output monitor to predict fluid responsiveness in mechanically ventilated children. Pediatr Cardiol. 2014;35(2):289-94.

15. da Silva Ramos FJ, Hovnanian A, Souza R, Azevedo LCP, Amato MBP, Costa ELV. Estimation of Stroke Volume and Stroke Volume Changes by Electrical Impedance Tomography. Anesth Analg. 2018;126(1):102-10.

16. Jozwiak M, Mercado P, Teboul JL, Benmalek A, Gimenez J, Depret F, et al. What is the lowest change in cardiac output that transthoracic echocardiography can detect? Crit Care. 2019;23(1):116.

17. Zlicar M, Novak-Jankovic V, Blagus R, Cecconi M. Predictive values of pulse pressure variation and stroke volume variation for fluid responsiveness in patients with pneumoperitoneum. J Clin Monit Comput. 2018;32(5):825-32.

18. Han D, Pan S, Wang X, Jia Q, Luo Y, Li J, et al. Different predictivity of fluid responsiveness by pulse pressure variation in children after surgical repair of ventricular septal defect or tetralogy of Fallot. Paediatr Anaesth. 2017;27(10):1056-63.

19. Han D, Liu Y-G, Luo Y, Li J, Ou-Yang C. Prediction of Fluid Responsiveness Using Pulse Pressure Variation in Infants Undergoing Ventricular Septal Defect Repair with Median Sternotomy or Minimally Invasive Right Thoracotomy. Pediatr Cardiol. 2016;38(1):184-90.

20. Zorio V, Lebreton T, Desgranges FP, Bochaton T, Desebbe O, Chassard D, et al. Does a two-minute mini-fluid challenge predict fluid responsiveness in pediatric patients under general anesthesia? Paediatr Anaesth. 2020;30(2):161-7.

21. Feld LG, Neuspiel DR, Foster BA, Leu MG, Garber MD, Austin K, et al. Clinical Practice Guideline: Maintenance Intravenous Fluids in Children. Pediatrics. 2018;142(6). 
22. Iyengar AJ, Winlaw DS, Galati JC, Celermajer DS, Wheaton GR, Gentles TL, et al. Trends in Fontan surgery and risk factors for early adverse outcomes after Fontan surgery: the Australia and New Zealand Fontan Registry experience. J Thorac Cardiovasc Surg. 2014;148(2):566-75.

23. Michard F, Teboul JL. Predicting fluid responsiveness in ICU patients: a critical analysis of the evidence. Chest. 2002;121(6):2000-8.

24. Yoshitake S, Miyamoto T, Tanaka Y, Naito Y. Non-invasive measurement of cardiac output using AESCULON((R)) mini after Fontan operation. Pediatr Int. 2017;59(2):141-4.

\section{Figures}

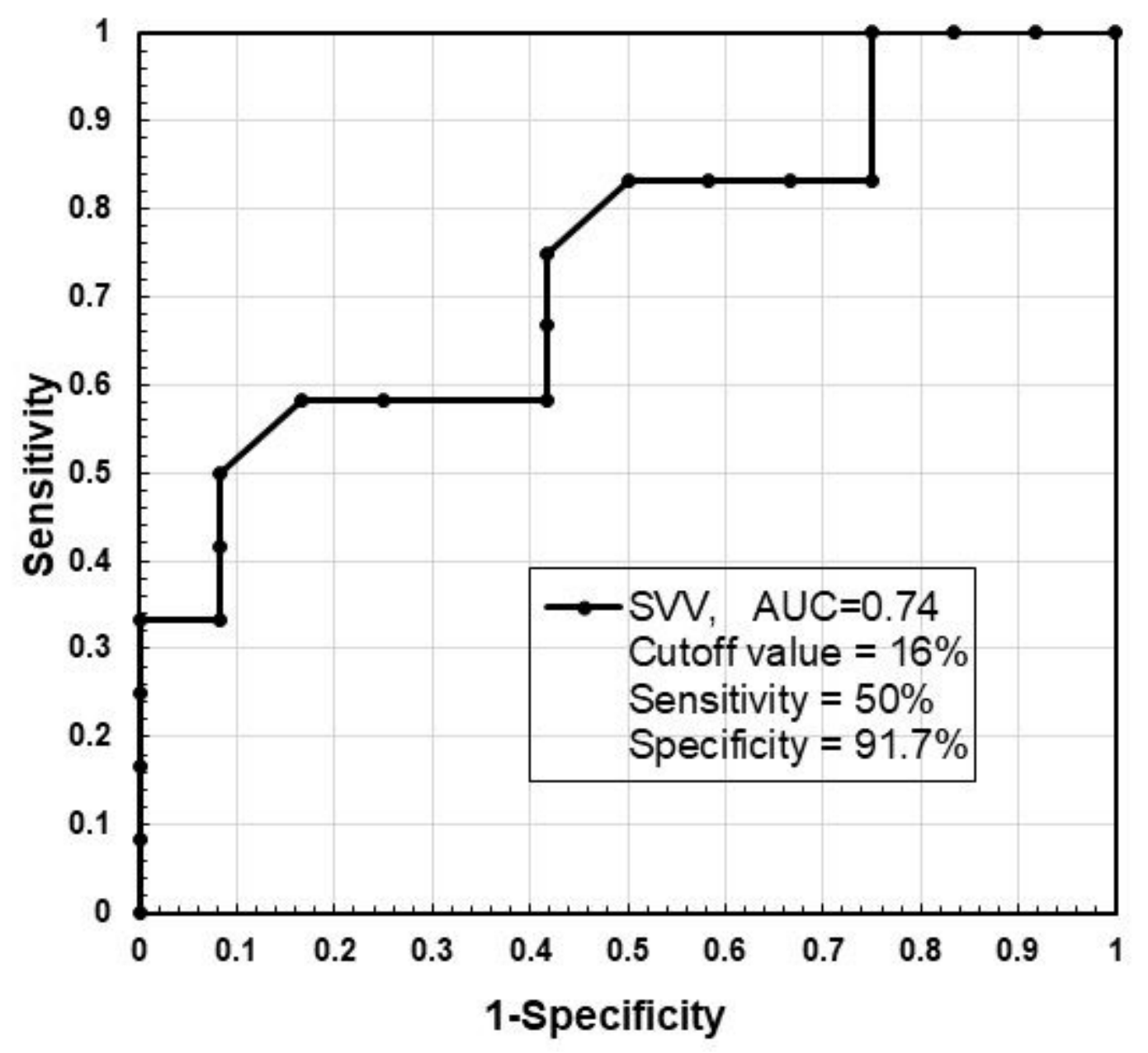

\section{Figure 1}

Receiver operating curves of SVV to predict fluid responsiveness in Fontan patients; AUC was 0.74 (95\% $\mathrm{Cl} 0.54-0.94, p<0.05$ ) and the cutoff value was $16 \%$, offering a sensitivity of $50 \%$ and a specificity of $91.7 \%$. 


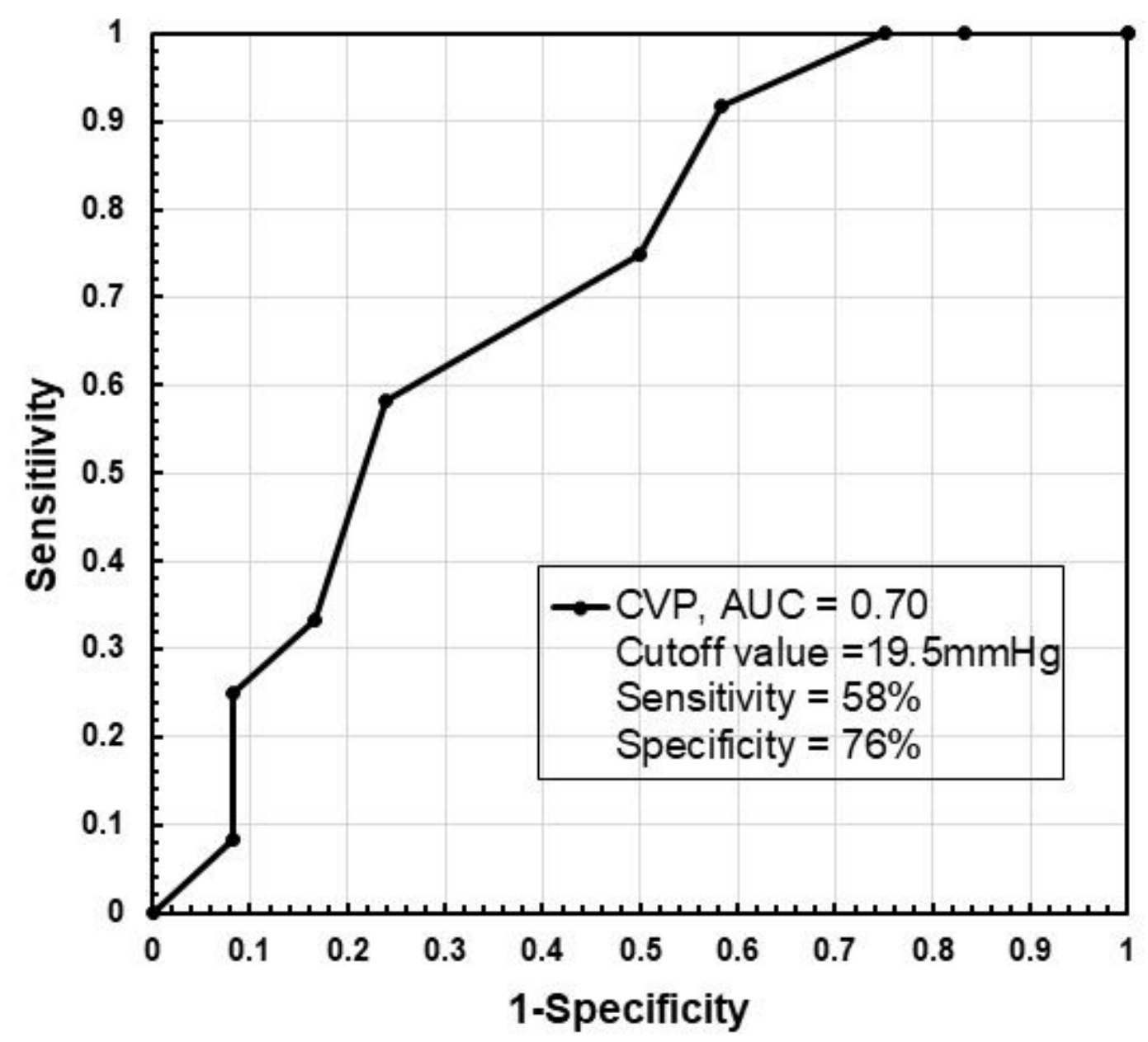

Figure 2

Receiver operating curves of CVP to predict fluid responsiveness in Fontan patients. AUC was 0.70 (95\% $\mathrm{Cl} 0.50-0.92, P>0.05)$ and the cutoff value was $19.5 \mathrm{mmHg}$, offering a sensitivity of $58 \%$ and a specificity of $76 \%$. 